\author{
D. Ferro • L. Loffredo • L. Polimeni • F. Violi
}

\title{
Underuse of oral anticoagulants in patients with nonvalvular atrial fibrillation in Italy
}

Received: 24 July 2006 / Accepted in revised form: 30 October 2006 / Published online: 31 March 2007

\begin{abstract}
Introduction: Oral anticoagulants (OAs) are significantly more effective than Aspirin in the prevention of cerebrovascular accidents among patients with atrial fibrillation (AF). Several studies, however, showed OAs to be widely underused in these patients. Objective: To assess the appropriateness of antithrombotic therapy in an Italian population of AF patients. Methods: Two hundred and fifty-five consecutive patients affected by nonvalvular AF participated in the study. Data were collected on demographic characteristics, risk factors for stroke, current prophylactic therapy, and perceived or actual risk factors for bleeding. INR levels were measured. Patients were stratified by their risk for stroke (214 at high risk, 21 moderate, 20 low), and their prophylactic therapy was analysed in light of international antithrombotic therapy recommendations. After therapy adjustment, 203 of our patients were followed-up for the occurrence of cerebrovascular events for an average of 27 months. Results: Upon admission, $35 \%(n=75)$ of patients in the high-risk category were either taking no antithrombotic prophylaxis or were being treated with Aspirin. In addition, 38 of 139 patients receiving OAs had an INR $<2$. Thus, a total of $113(52.8 \%)$ high-risk subjects were not receiving adequate antithrombotic therapy. Of high-risk patients not treated with OAs, $46.7 \%$ reported no perceived
\end{abstract}

D. Ferro $\bullet$ L. Loffredo $\bullet$ L. Polimeni $\bullet$ F. Violi

Dipartimento di Medicina Sperimentale e Patologia

Università degli Studi "La Sapienza"

Rome, Italy

D. Ferro (ه)

IV Clinica Medica

Policlinico Umberto I

Rome, Italy

e-mail: mimmo.ferro@virgilio.it or actual risk factors for bleeding. The annual incidence of cerebrovascular accidents was 3.8\% among 163 high-risk patients assigned to OA treatment, and $4.5 \%$ among 39 patients given Aspirin treatment. Relative to expected annual incidence rates, cerebrovascular risk in anticoagulated patients was reduced by about $70 \%$. Conclusions: Underuse of OAs is still common in Italy, and much of it cannot be explained by the concern for haemorrhage. Support and training in the complex task of anticoagulation management may help to extend this efficacious prophylactic therapy to all patients who may benefit from it.

Keywords Atrial fibrillation • Oral anticoagulants • Ischaemic stroke

\section{Introduction}

Atrial fibrillation (AF) is a common disorder of cardiac rhythm and a strong, independent risk factor for stroke, as it is associated with the formation of left atrial thrombi [1]. With advancing age, AF becomes an increasingly important cause of stroke, as the prevalence of this arrythmia rises from $<1 \%$ in those less than 60 years old to $8 \%-10 \%$ in those older than 80 [2-4].

Adjusted-dose warfarin was found to reduce the incidence of stroke by about $60 \%$, with absolute risk reductions of $3 \%$ per year for primary prevention (referring to patients without previous stroke or transient ischaemic attacks (TIAs)) and $8 \%$ per year for secondary prevention (referring to patients with previous stroke or TIA) [5-7]. In contrast, Aspirin reduced the incidence of stroke by about $20 \%$, with absolute risk reductions of $1.5 \%$ per year for primary prevention and $2.5 \%$ per year for secondary prevention [8]. When compared to Aspirin, warfarin - at a dose adjusted to maintain an International Normalised Ratio (INR) of 2-3 reduced the risk by about $40 \%$, with similar relative risk 
reductions for primary and secondary prevention [9]; Aspirin seems to be efficacious mainly in the prevention of smaller, noncardioembolic strokes $[1,10]$.

Despite strong evidence in support of the clinical efficacy of oral anticoagulants (OAs), and the lack of contraindications to their use, underuse of these medications is quite common. In fact, anticoagulants are given to less than $50 \%$ of AF patients at highest risk for stroke [11-13].

The aim of this study was to evaluate the appropriateness of antithrombotic therapy in an Italian population of $\mathrm{AF}$ patients. To reach this objective, we stratified patients by their risk for stroke, and analysed the therapy they were receiving on admission, in light of international antithrombotic therapy recommendations for patients in their risk category. Most patients were then followed up for the occurrence of stroke or TIA after therapy adjustment.

\section{Methods}

Two hundred and fifty-five eligible subjects were recruited, between April 2001 and September 2005, among consecutive patients admitted to the IV Division of Clinical Medicine due to nonvalvular AF - paroxysmal, persistent or permanent (lasting $>6$ months). Exclusion criteria were: mitral stenosis, mitral valve prosthesis or severe mitral regurgitation, lone AF, cancer, liver or kidney diseases, acute or chronic inflammatory disorders.

Of the 255 patients recruited, 191 (74.9\%) had permanent or persistent $\mathrm{AF}$, and $64(25.1 \%)$ had paroxysmal AF.

Upon admission, the clinical staff interviewed patients and reviewed their medical charts. The following data were collected: demographics; actual or perceived risk factors for bleeding or thromboembolic events; curative and prophylactic treatment for thromboembolic and ischaemic events.

In order to assess the correct anticoagulation required and the quality of anticoagulation control, two INR measurements were performed on each patient at baseline, one week apart. Anticoagulation intensity was considered insufficient if INR was $<2$ on both occasions.

Patients were stratified by their risk for stroke into the three categories below [1]:

High risk: patients with previous TIA or cerebrovascular accident; patients aged $\geq 75$ with diabetes or hypertension; patients with clinical evidence of valve disease, heart failure, thyroid disease and impaired left ventricular function.

Moderate risk: patients aged $<65$ with clinical risk factors: diabetes, hypertension, peripheral vascular disease, ischaemic heart disease; patients $>65$ not in the high-risk group.

Low risk: patients aged $<65$ with no history of embolism, hypertension, diabetes or other clinical risk factors.

For the three risk categories $(n=255)$, we calculated the proportions of patients by type of antithrombotic prophylaxis received on admission (OAs, Aspirin, none), and the proportion of patients with insufficient anticoagulation intensity.

Two hundred and three of the patients were also followed up for the incidence of stroke or TIA, for an average of 27 months (range 6-36 months). Prophylactic therapy was reconsidered for all subjects before the beginning of follow-up. During follow-up, OA doses were regularly adjusted based on periodic INR measurements, to maintain INR levels of 2-3. The occurrence of stroke was determined on the basis of clinical manifestations, and confirmed by computed tomography.

\section{Results}

Clinical characteristics of the entire study population $(n=255)$ are reported in Table 1. Mean age was 72.6 years, with $44.7 \%$ of subjects older than 75 . Forty-seven percent were male. Hypertension was the most frequently reported risk factor for stroke $(78.8 \%)$, followed by coronary artery disease $(31.8 \%)$, congestive heart failure $(31.4 \%)$ and diabetes $(16.1 \%) ; 24.3 \%$ of patients reported a history of stroke, TIA or systemic embolic event.

About $73 \%$ of patients were receiving antihypertensive treatment (one or more medication): diuretics (58.1\%), ACEinhibitors $(54.6 \%)$, calcium-antagonists $(47.1 \%)$ and betablockers $(29.7 \%)$.

Of the 255 patients studied, $84 \%$ were stratified to the high risk group, $8.2 \%$ to the moderate risk group and $7.8 \%$ to the low risk group. Figure 1 shows prophylactic therapy on admission by risk group: among patients at low risk, $34.5 \%$ were being treated with OAs, $41.5 \%$ were being treated with Aspirin and $24 \%$ were taking no medications. Among patients at high risk, $65 \%(n=139)$ were being treated with OAs, but 38 of these subjects were insufficiently anticoagulated $(\mathrm{INR}<2)$. In addition, 35\% $(n=75)$ of high-

Table 1 Patient characteristics $(N=255)$

\begin{tabular}{ll}
\hline Demographic characteristics & Frequency $(\%)$ \\
\hline $\begin{array}{l}\text { Mean age (years } \pm \text { SD) } \\
\text { Age }\end{array}$ & $72.6 \pm 10.4$ \\
$\quad+65$ & $51(20.0 \%)$ \\
$66-75$ & $90(35.3 \%)$ \\
$>75$ & $114(44.7 \%)$ \\
Gender & \\
Male & $120(47.0 \%)$ \\
Female & $135(53.0 \%)$ \\
Clinical characteristics & \\
Hypertension & $201(78.8 \%)$ \\
CAD/atherosclerosis & $81(31.8 \%)$ \\
CHF (LV dysfunction) & $80(31.4 \%)$ \\
Diabetes & $41(16.1 \%)$ \\
History of stroke, TIA or systemic embolus & $62(24.3 \%)$ \\
History of AMI & $46(18 \%)$ \\
History of DVT or PE & $11(4.3 \%)$ \\
Thyrotoxicosis & $2(0.8 \%)$ \\
Behavioural risk factors & \\
Cigarette smoking & $48(18.8 \%)$ \\
Alcohol abuse & $51(20 \%)$ \\
\hline
\end{tabular}

$C A D$, coronary artery disease; $C H F$, congestive heart failure; $L V$, left ventricular; TIA, transient ischaemic attack; $D V T$, deep vein thrombosis; $P E$, pulmonary embolism 


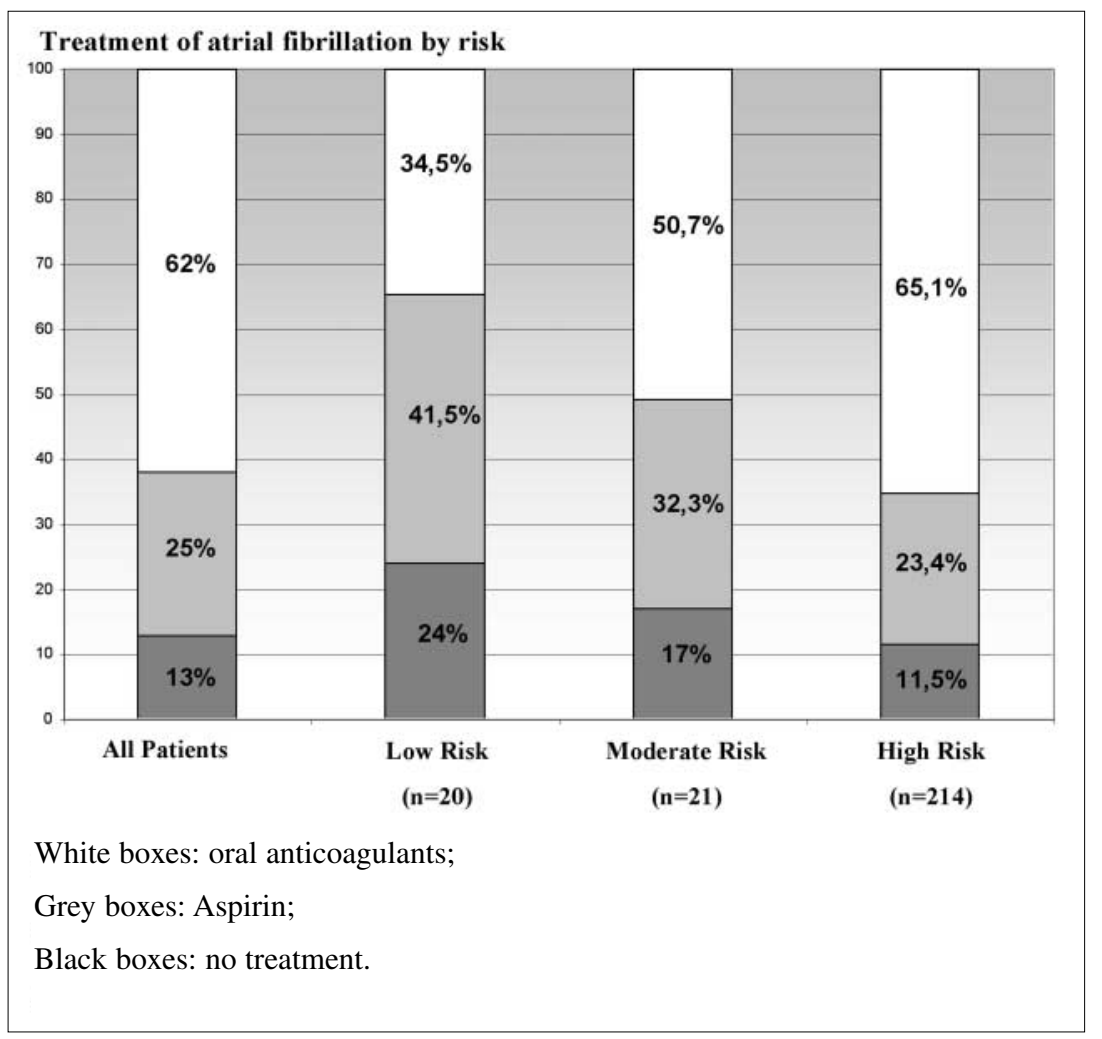

Fig. 1 Antithrombotic prophylaxis in AF patients (\%) by risk category risk patients were either not receiving prophylaxis $(11.5 \%)$ or were being treated with Aspirin (23.4\%). Considering the entire group of patients at high risk, 113 subjects $(52.8 \%)$ were not receiving adequate antithrombotic therapy.

Patients with persistent or permanent AF were more likely to be treated with OAs than those with paroxysmal AF (67\% vs. 52\%; $p<0.05)$.

To explore possible reasons for not prescribing anticoagulation therapy, we analysed data on perceived or actual risk factors for bleeding among patients at high risk for stroke who were not receiving OAs $(n=75)$. Neuropsychological impairment was reported in $36 \%$ of cases, risk of falling in $22.7 \%$, a past bleeding episode in $16 \%$, peptic ulcer in $12 \%$ and a history of aneurysm in $4 \%$. Almost $48 \%$ of patients reported no risk factors for haemorrhage (Table 2).

Two hundred and three patients (95 males, 108 females, mean age $71.9 \pm 10.73$ years) participated in the follow-up.

Table 2 Factors associated with perceived or actual risk of bleeding among high-risk patients not receiving OA therapy $(N=75)$

\begin{tabular}{ll}
\hline Factor & Frequency $n(\%)^{*}$ \\
\hline Neuropsychological impairment & $27(36.0)$ \\
Risk of falling & $17(22.7)$ \\
Past bleeding episode & $12(16.0)$ \\
Peptic ulcer disease & $9(12.0)$ \\
History of aneurysm & $3(4.0)$ \\
None of these factors & $35(46.7)$ \\
\hline
\end{tabular}

*Some patients reported more than one factor
Eighty-eight percent of them were stratified to the high-risk category, $7 \%$ to the moderate-risk category and $5 \%$ to the low-risk category.

We prescribed OAs to 163 of 178 followed-up high-risk patients. The remaining 15, who had poor compliance for $\mathrm{OA}$, as well as all 14 moderate-risk patients and 10 of 11 low-risk patients, were given Aspirin. One low-risk patient refused any treatment.

During follow-up, 14 of 163 patients on OA (8.6\%) experienced a cerebrovascular accident (12 stroke, 2 TIA), with an annual incidence of $3.8 \%$, compared to 4 of 39 patients treated with Aspirin (10.2\% - 1 stroke, 3 TIA), with an annual incidence of $4.5 \%$. If the expected annual risk of cerebrovascular accident among untreated AF patients at high risk ranges between 8 and $12 \%$ [1], treatment with OAs reduced it by up to $70 \%$. In contrast, in patients with moderate or low risk, whose expected annual risk of cerebrovascular event is $1 \%-4 \%$ [1], treatment with Aspirin did not seem to affect the annual rate of ischaemic events.

\section{Discussion}

Our data indicate that an underuse of OAs may be detected in $35 \%$ of high-risk patients in an Italian population with nonvalvular AF.

Several AF trials have shown that OAs are highly effective in the prevention of stroke and death caused by thromboem- 
bolism. Based on these studies, guidelines for the use of OAs in AF patients at risk of stroke (high- and moderate-risk categories) have been published and are widely accepted [14-19].

In clinical practice however, the actual use of anticoagulation in patients at risk for stroke has been disappointingly low. Despite great variability between studies, with appropriate antithrombotic therapy rates ranging from $15 \%$ to $79 \%$ [13], in most studies, appropriate OA treatment is observed only in about $50 \%$ of cases [19-23].

In a recent study covering a broad geographic cross section of US hospitals, the overall proportion of warfarin use was 54\%, 25\% were treated with Aspirin and 22\% received no medication [23]. Compared to these data, our study, performed using similar risk stratifications, shows a more frequent use of OAs. In fact, among our high-risk patients, $65 \%$ were taking OAs, $23.4 \%$ were being treated with Aspirin and only $11.5 \%$ were left untreated. Indeed, the use of OAs in AF patients has increased in Italy in the past few years, possibly thanks to the establishment of anticoagulation clinics in our country, a fruit of efforts that began with the founding of the Italian Federation of Centers for the Surveillance of Anticoagulant Therapies in 1989 [24].

Yet OA treatment, even where given, is not always adequate: in our study, $27.3 \%$ of patients treated with OAs on admission had an INR $<2$, indicating insufficient anticoagulation intensity. Taking together high-risk patients who were not being treated with OAs, and those with insufficient anticoagulation intensity, $52.8 \%$ of our high-risk patients were not receiving the treatment recommended by international guidelines.

Several surveys have studied physicians' barriers to prescribing anticoagulant therapy. Practical barriers to the use of OAs include the need for frequent blood testing for prothrombin time and ongoing dose adjustments [25, 26].

Patient-related factors such as old age, perceived embolic risk and perceived risk of haemorrhage have been repeatedly found to influence decisions on OA treatment [27-30]. The reluctance to prescribe dose-adjusted OAs to elderly patients is of special concern in view of the fact that trials show these patients benefit most from this therapy [31, 32]. Surveyed physicians have reported withholding OA therapy based on the belief that patients would refuse therapy or be noncompliant. According to one survey, $79 \%$ of physicians cited a lack of patient reliability as a contraindication to therapy [29].

In a clinical scenario depicting a patient with a history of falls, $71 \%$ of physicians believed anticoagulation therapy was contraindicated [28]. Finally, some physicians reported difficulty in maintaining INR levels within the therapeutic range $[25,33]$.

Our data show however, in agreement with a recent study [23], that among high-risk patients not treated with OAs, about one half report none of the factors associated with perceived or actual risk factors for bleeding. This indicates that much OA underuse may not be accounted for by a concern for haemorrhage.
An interesting finding in this context is the fact that paroxysmal AF patients seem to be significantly less likely to be treated with OAs than patients with persistent or permanent AF. This phenomenon, observed in our population as well as in another recent report [23], may point to a tendency among some physicians to underestimate the risk of stroke in paroxysmal AF - a risk that is similar to that of patients with persistent or permanent AF.

These and other results, indicating that urban patients are more likely to receive anticoagulant therapy compared to rural subjects ( $58 \%$ vs. $47 \%$ ), and that cardiologists tend to prescribe it more often than noncardiologists (69\% vs. 52\%) [21], suggest that support and training in the complex task of anticoagulation management may help extend and improve the use of OA prophylaxis in AF patients at risk of cerebrovascular events.

At the start of follow-up, we reconsidered antithrombotic therapy and assigned OAs to all but 15 patients at high risk of cerebrovascular accident. After 27 months of follow-up, a correct use of OAs was able to reduce the risk of ischaemic stroke by about $70 \%$. These results are in agreement with those of randomised, controlled trials showing that doseadjusted anticoagulation reduces the risk of ischaemic stroke by about $68 \%$ in unselected patients with AF [5-7].

In contrast, among patients with moderate or low risk, treatment with Aspirin did not seem to reduce the annual rate of ischaemic events. This may be due to a limited capacity of this type of treatment to prevent serious, cardioembolic events, which are frequent in AF patients $[1,10]$.

The small number of subjects, especially in the low and moderate risk categories, is a limitation of the present study. Larger, multicentre studies will be needed to confirm our findings, and help clarify the reasons underlying OA prescription practices in Italy.

In conclusion, this study shows that about one half of Italian AF patients at high risk for stroke either did not receive OAs or were receiving insufficient anticoagulation. Support and training in the complex task of anticoagulation management, as well as improved communication between all those involved - patients, physicians and OA centres may help offer AF patients better protection from cerebrovascular accidents through correct prophylactic anticoagulation therapy.

\section{References}

1. Lip GYH, Hart RG, Conway DSG (2002) Antithrombotic therapy for atrial fibrillation. BMJ 325:1022-1025

2. Albers GW, Dalen JE, Laupacis A et al (2001) Antithrombotic therapy in atrial fibrillation. Chest 119:194S-206S

3. Fuster V, Ryden LE (2001) ACC/AHA/ESC guidelines for the management of patients with atrial fibrillation. J Am Coll Cardiol 38:1231-1266 
4. Wolf PA, Abbott RD, Kannel WB (1987) Atrial fibrillation: a major contributor to stroke in the elderly. The Framingham Study. Arch Intern Med 147:1561-1564

5. - (1994) Risk factors for stroke and efficacy of antithrombotic therapy in atrial fibrillation. Analysis of pooled data from five randomized controlled trials. Arch Intern Med 154:1449-1457

6. Hart RG, Benavente O, McBride R, Pearce LA (1999) Antithrombotic therapy to prevent stroke in patients with atrial fibrillation: a meta-analysis. Ann Intern Med 131:492-501

7. Hart RG, Halperin JL, Pearce LA et al (2003) Lessons from the stroke prevention in atrial fibrillation trials. Ann Intern Med 138:831-839

8. Petersen P, Boysen G, Godtfredsen J, Andersen ED, Andersen B (1989) Placebo-controlled, randomized trial of warfarin and aspirin for prevention of thromboembolic complications in chronic atrial fibrillation. The Copenhagen AFaSAK study. Lancet 1:175-179

9. - (1994) Warfarin versus aspirin for prevention of thromboembolism in atrial fibrillation: Stroke Prevention in Atrial Fibrillation II study. Lancet 343:687-691

10. Hart RG, Pearce LA, Miller VT et al (2000) Cardioembolic vs. noncardioembolic strokes in atrial fibrillation: frequency and effect of antithrombotic agents in the stroke prevention in atrial fibrillation studies. Cerebrovasc Dis 10:39-43

11. Cohen N, Almoznino-Sarafian D, Alon I et al (2000) Warfarin for stroke prevention still underused in atrial fibrillation. Patterns of omission. Stroke 31:1217-1222

12. Bungard TJ, Ghali WA, Teo KK, McAlister FA, Tsuyuki RT (2000) Why do patients with atrial fibrillation not receive warfarin? Arch Intern Med 160:41-46

13. Buckingham TA, Hatala R (2002) Anticoagulants for atrial fibrillation: why is the treatment so low? Clin Cardiol 25:447-454

14. - (1991) Stroke Prevention in Atrial Fibrillation study. Final results. Circulation 84:527-529

15. - (1996) Adjusted-dose warfarin versus low-intensity, fixeddose warfarin plus aspirin for high-risk patients with atrial fibrillation: Stroke Prevention in Atrial Fibrillation III randomized clinical trial. Lancet 348:633-638

16. - (1990) The Boston Area Anticoagulation Trial for Atrial Fibrillation Investigators. The effect of low-dose warfarin on the risk of stroke in patients with nonrheumatic atrial fibrillation. N Engl J Med 323:1505-1511

17. Ezekowitz MD, Bridgers SL, James KE et al (1992) Warfarin in the prevention of stroke associated with nonrheumatic atrial fibrillation. N Engl J Med 327:1406-1412

18. Brophy MT, Snyder KE, Gaedhe S et al (2004) Anticoagulant use for atrial fibrillation in the elderly. J Am Geriatr Soc 52:1151-1161

19. Rockson SG, Albers GW (2004) Comparing the guidelines: anticoagulation therapy to optimize stroke prevention in patients with atrial fibrillation. J Am Coll Cardiol 43:929-935

20. Adhiyaman V, Kamalakannan D, Oke A, Shah IU, White AD (2000) Underutilization of antithrombotic therapy in atrial fibrillation. J R Soc Med 93:138-140

21. Flaker GC, McGowan DJ, Boechler M, Fortune G, Gage B (1999) Underutilization of antithrombotic therapy in elderly rural patients with atrial fibrillation. Am Heart J 137:307-312

22. Mendelson G, Aronow WS (1998) Underutilization of warfarin in older persons with chronic nonvalvular atrial fibrillation at high risk for developing stroke. J Am Geriatr Soc 46:1423-1424

23. Waldo AL, Becker RC, Tapson VF, Colgan KJ for the NABOR Steering Committee (2005) Hospitalized patients with atrial fibrillation and a high risk of stroke are not being provided with adequate anticoagulation. J Am Coll Cardiol 46:1729-1736

24. Berrettini M (1997) Anticoagulation clinics: the Italian experience. Haematologica 82:713-717

25. Kutner M, Nixon G, Silverstone F (1991) Physicians' attitudes toward oral anticoagulants and antiplatelet agents for stroke prevention in elderly patients with atrial fibrillation. Arch Intern Med 151:1950-1953

26. McCrory DC, Matchar DB, Samsa G, Sanders LL, Pritchett ELC (1995) Physician attitudes about anticoagulation for nonvalvular atrial fibrillation in the elderly. Arch Intern Med 155:277-281

27. Whittle J, Wickenheiser L, Venditti LN (1997) Is warfarin underused in the treatment of elderly persons with atrial fibrillation? Arch Intern Med 157:441-445

28. Monette J, Gurwitz JH, Rochon PA, Avorn J (1997) Physician attitudes concerning warfarin for stroke prevention in atrial fibrillation: results of a survey of long- term care practitioners. J Am Geriatr Soc 45:1060-1065

29. Chang HJ, Bell JR, Devoo DB, Kirk JW, Wasson JH (1990) Physician variation in anticoagulating patients with atrial fibrillation. Arch Intern Med 150:81-84

30. Lip GYH, Zarifis J, Watson RDS, Beevers DG (1996) Physician variation in the management of patients with atrial fibrillation. Heart 75:200-205

31. Copland M, Walker ID, Campbell Tait RC (2001) Oral anticoagulation and hemorrhagic complications in an elderly population with atrial fibrillation. Arch Intern Med 161:2125-2128

32. Man-Son-Hing M, Laupacis A (2003) Anticoagulant-related bleeding in older persons with atrial fibrillation. Physicians' fears often unfounded. Arch Intern Med 163:1580-1586

33. Rodgers H, Sudlow M, Dobson R, Kenny RA, Thomson RG (1997) Warfarin anticoagulation in primary care: a regional survey of present practice and clinicians' views. Br J Gen Pract 47:309-310 